\title{
Clinical Study \\ Clinicopathological Proficiency in the Diagnosis of Kaposi's Sarcoma
}

\author{
Louis-Jacques van Bogaert \\ National Health Laboratory Service, Polokwane/Mankweng Hospital Complex and University of Limpopo, \\ Limpopo Province, 0700 Polokwane, South Africa
}

Correspondence should be addressed to Louis-Jacques van Bogaert, louis.vanbogaert@nhls.ac.za

Received 21 March 2012; Accepted 9 April 2012

Academic Editors: K. Gee, L. Gray, and C. Petrovas

Copyright () 2012 Louis-Jacques van Bogaert. This is an open access article distributed under the Creative Commons Attribution License, which permits unrestricted use, distribution, and reproduction in any medium, provided the original work is properly cited.

\begin{abstract}
Background. The prevalence of Kaposi's sarcoma (KS), an AIDS-defining illness, has increased in parallel with the HIV/AIDS epidemic. The presence of violaceous skin lesions should raise suspicion of KS. However, especially on dark skin, KS mimics a variety of non-KS skin conditions. Histologically, there is a wide range of expressions of KS and a large number of mimickers. For all these reasons, a HHV-8 immunohistochemically biopsy-proven diagnosis of KS should be the gold standard. Methods. Prospective study of 490 consecutive skin biopsies from the general community in the Limpopo Province of South Africa, from April 2010 through December 2011. Results. The clinical discordance rate (over-/underdiagnosis of KS) was 30.5\%; the histological discordance rate was $9.2 \%$. Conclusion. Because of the magnitude of diagnostic error, both clinical and histological, all clinical lesions suspicious of KS should be biopsied and HHV-8 LAN-1 immunophenotyped.
\end{abstract}

\section{Introduction}

Kaposi's sarcoma (KS) became an AIDS-defining illness at the outset of the human-immunodeficiency-virus-(HIV-) induced acquired immunodeficiency syndrome (AIDS) endemic and pandemic [1-3]. There has been a striking increase in incidence of KS in both men and women compatible with the evolution of the AIDS epidemic in sub-Saharan Africa $[4,5]$. In HIV/AIDS endemic regions such as subSaharan Africa, purplish/violaceous skin lesions should arise a high degree of awareness and suspicion of KS. However, the clinical picture of KS may mimic a variety of non-KS lesions. Furthermore, the histopathological expression of KS encompasses a large number of mimics too [6]. Finally, not all KS are HIV-related [3].

Sub-Saharan Africa in general and South Africa in particular are at the epicenter of the HIV/AIDS endemic. Although the prevalence rates vary somehow according to the sources, it was estimated that in 2010 it infected 17.8 percent of the South African population and up to 19.7 percent of females aged between 15 and 45 years [7]. Because of the opt-in policy for testing (voluntary counseling and testing) and the arguable widespread risk of discrimination after disclosure of positivity necessary for access to antiretroviral treatment (ART), an undefined portion of the population's HIV status remains undiagnosed, unknown, or undisclosed [8].

The human herpes virus-8 (HHV-8) or Kaposi's sarcoma associated herpes virus (KSHV) was identified as the infectious agent of all types of KS (classic/sporadic, iatrogenic/ posttransplant, endemic/African, and AIDS-related/HIVassociated) [9]. The latent nuclear antigen-1 (LAN-1) of HHV-8 is expressed in all cells infected by the virus [10]. The immunohistochemical identification of HHV-8 by LAN1 has become the "gold standard" in the diagnosis of KS and the differential diagnosis with its mimics. It has been shown to have a high specificity and sensitivity for the diagnosis of KS [11-14].

Because of prognostic and therapeutic implications of a correct diagnosis, it is recommended to biopsy any skin lesion susceptible of being KS, especially in HIV/AIDS endemic regions. We report an observational cohort of 490 consecutive skin biopsies in HIV-infected and noninfected 
TABle 1: Age distribution by gender, HIV serostatus, and Kaposi's sarcoma.

\begin{tabular}{lcccc}
\hline & Male & Female & $t$ & $P$ \\
\hline HIV seropositive & $41.6 \pm 12.6[41.0]^{*}$ & $40.5 \pm 10.7[38.0]$ & 0.46 & 0.15 \\
HIV status unknown & $41.7 \pm 18.0[43.0]$ & $41.1 \pm 18.8[39.0]$ & 0.21 & 0.84 \\
Biopsy-proven KS & $41.1 \pm 14.1[40.0]$ & $42.2 \pm 15.7[39.5]$ & 0.54 & 0.59 \\
\hline
\end{tabular}

${ }^{*}$ Values are mean $\pm \mathrm{SD}[$ median].

patients. All biopsies were immunostained with HHV-8 LANA-1; 261 (53.3\%) were biopsy-proven cases of KS among a group comprising 248 (50.6\%) HIV-infected patients. We investigated the rates of clinical and histological diagnostic proficiency of KS and of its clinical and histopathological mimics.

\section{Methods}

2.1. Site and Population. The study was carried out in the Limpopo Province of South Africa. All surgical pathology specimens of public health facilities are centralized at the National Health Laboratory Service in the provincial capital, Polokwane. It serves an essentially rural population in excess of 5 Million. The study was approved by the research ethics committee. All the cases were treated anonymously.

2.2. Data Collection. The cases were collected from April 2010 through December 2011. All the patients were of African ethnicity. The following information was recorded: age, gender, HIV status (when available), ART status, CD4+ T lymphocyte count, site of biopsy, and clinical provisional diagnosis. Only 25 biopsies were referred by specialist dermatologists.

All of the cases were routinely streptavidin-biotinperoxidase immunostained with diaminobenzidine using a murine monoclonal antibody directed against the Cterminus of the LAN-1 molecule of HHV-8 (clone 13b10; Novocastra, New Castle upon Tyne, UK). The initial histopathological diagnosis was made before LAN-1 immunostaining and corrected accordingly when indicated in retrospect. The clinical and histopathological diagnostic proficiency was expressed in terms of concordance or discordance. Discordance was further subdivided into overdiagnosis (non-KS) and underdiagnosis (KS missed).

Statistical analysis was performed using column statistics, Student's $t$ test, and $95 \%$ confidence intervals (CI) of proportions. The level of significance was set at $P<0.05$.

\section{Results}

Out of 490 cases, 261 (53.3\%) were HHV-8 proven cases of KS. The rate of documented HIV positivity among the 490 cases was 248 (50.6\%). Eighty-one (32.7\%) were documented to be on ART and $24(9.7 \%)$ not to be on ART. The average CD4++ count was $259.0 \pm 181.6$ cells $/ \mathrm{mm}^{3}$ (median: 214.0; range: $6.0-674$ ).

Among the 261 cases of KS, 161 (61.7\%) were known to be HIV-infected; no HIV serostatus was known for the 100 others. Among the KS with unknown HIV status, 52 were males and 48 females. One KS only was HIV negative at the time of biopsy.

Table 1 shows the average ages by gender, HIV status, and diagnosis of KS. There was no statistically significant difference between the groups.

The anatomical site of KS, illustrated in Table 2, was known in $212(81.2 \%)$ cases. The lower limbs were affected in $43.4 \%$; the lesions were disseminated in $29.7 \%$. The head was significantly involved in the HIV unknown serostatus, while dissemination was significantly more common in the documented HIV-infected patients.

Table 3 lists the distribution by histopathological stage. There was no significant difference in distribution of stages between the patients with unknown HIV serostatus and documented HIV-positive cases.

Table 4 illustrates the biopsy-proven non-KS skin lesions according to the HIV status. Pyogenic granuloma and seborrheic keratosis represented close to half of the non-KS lesions.

The histological rate of discordance, shown in Table 5, was $9.2 \%$; $18 \mathrm{KS}$ were missed, and 23 non-KS were overdiagnosed. Fibrous histiocytoma $(n=9)$, pyogenic granuloma $(n=5)$, and seborrheic keratosis $(n=8)$ were misdiagnosed as nodular stage and early patch KS, respectively.

Figure 1 shows the flow chart indicating that no clinical provisional diagnosis was provided in 49 (18.8\%) biopsyproven KS, and in 120 (52.4\%) of non-KS skin lesions. The total clinical discordance rate was 30.5\%; in $48(15.0 \%)$ with provisional clinical diagnosis, KS was overdiagnosed, and, in 59 (18.4\%), the diagnosis of KS was missed clinically. Seborrheic keratosis and pyogenic granuloma accounted for half of the total clinical discordance rate.

\section{Discussion}

Kaposi's sarcoma is a multifaceted entity in terms of the population groups (ethnic and gender) it affects, in terms of clinical signs and symptoms, in terms of the body parts it affects (skin, mucosa, lymph nodes, viscera), and in terms of histopathological expression. Traditionally, the six histological variants are the early patch stage, the nodular stage, the plaque stage, the intravascular, the lymphangiomalike, and the angiosarcoma-like type [15]. Recently, more than ten additional variants have been described $[16,17]$.

In sub-Saharan Africa, KS accounts for about $10.0 \%$ of HIV-related malignancies and may be the first manifestation of HIV [18]. As such, the diagnosis of KS may in many instances serve as an entry into the treatment circuit, provided consent is given for HIV testing. Patients with 


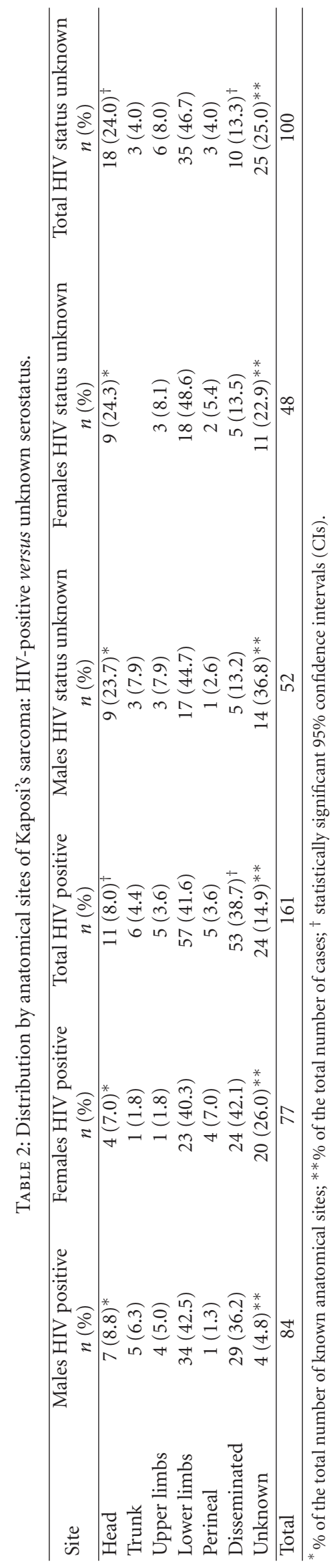




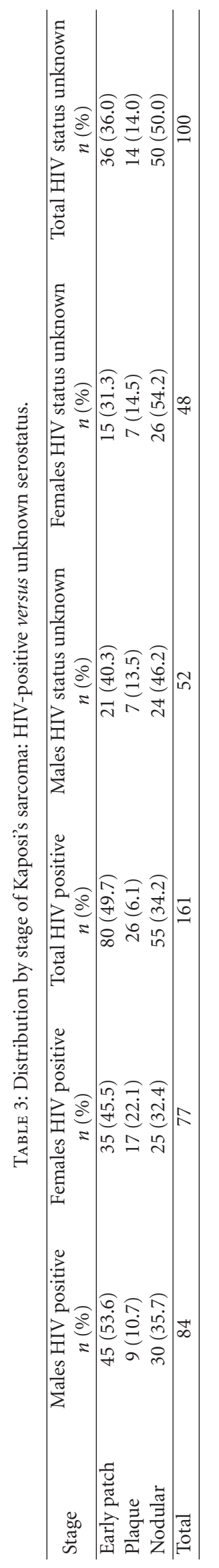


TABLE 4: Biopsy-proven non-Kaposi’s sarcoma skin lesions and HIV status.

\begin{tabular}{lccc}
\hline Pathology & HIV seropositive $n(\%)$ & HIV serostatus unknown $n(\%)$ & Total $n(\%)$ \\
\hline Pyogenic granuloma & $24(27.6)$ & $39(27.5)$ & $63(27.5)$ \\
Seborrheic keratosis & $29(33.3)$ & $22(15.5)$ & $51(22.3)$ \\
Haemangioma & $4(4.6)$ & $33(23.2)$ & $37(16.2)$ \\
Fibrous histiocytoma & $4(4.6)$ & $21(14.8)$ & $25(10.9)$ \\
Drug reaction vasculitis & $5(5.7)$ & $1(0.7)$ & $6(2.6)$ \\
Melanocytic melanoma & $3(3.4)$ & $2(1.4)$ & $5(2.2)$ \\
Varia & $18(20.8)$ & $24(16.9)$ & $42(18.3)$ \\
\hline Total & 87 & 142 & 229 \\
\hline
\end{tabular}

TABle 5: Histological diagnostic discordance before immunohistochemistry.

\begin{tabular}{lccr}
\hline Pathology & Overdiagnosis of KS $n(\%)$ & Underdiagnosis of KS $n(\%)$ & Total discordance rate $n(\%)$ \\
\hline Seborrheic keratosis & $8(29.6)$ & $7(38.9)$ & $15(33.3)$ \\
Fibrous histiocytoma & $9(33.3)$ & $3(16.7)$ & $12(26.7)$ \\
Pyogenic granuloma & $5(18.5)$ & $5(27.8)$ & $10(22.2)$ \\
Drug reaction vasculitis & $3(11.1)$ & $3(16.7)$ & $6(13.3)$ \\
Haemangioma & $2(7.4)$ & & $2(4.4)$ \\
\hline Total & 27 & 18 & $41 / 490(9.2)$ \\
\hline
\end{tabular}

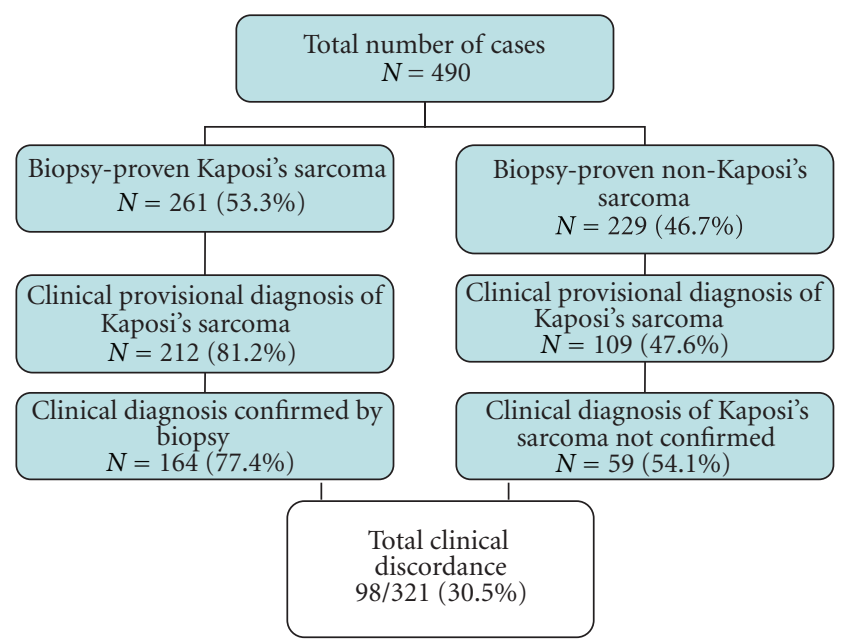

FIgURE 1: Flow chart of the clinical diagnostic concordance and discordance with histological diagnosis.

AIDS-associated KS present mostly with advanced disease resulting in high mortality [19]. Although KS regresses under ART, the relatively wide unavailability of ART, and the threshold of a CD4+ count of $\leq 200$ in South Africa as entry criterion into the ART circuit result in a rapid progression of the disease $[20,21]$. This illustrates not only the importance of an early and correct diagnosis of the disease but also of the willingness to undergo HIV testing.

Clinically, virtually all pigmented skin lesions are part of the differential diagnosis of KS [22]. In black subjects, the clinical recognition of $\mathrm{KS}$ is likely to be even more difficult than in nonblack subjects. This underscores the need of a biopsy-proven diagnosis. Because of the vast spectrum of vasoproliferative mimics of KS illustrated in Table 6,
HHV-8 LAN-1 immunohistochemistry has become the gold standard of diagnosis [16, 22-24].

The increasing prevalence of HIV infection in subSaharan Africa together with the reluctance to be tested makes it difficult to distinguish the endemic KS from the AIDS-associated variant [5]. The distinction has prognostic implications since endemic KS evolves much slower and less aggressively than the AIDS-associated variant. In a Tanzanian study of 105 clinically suspected KS, 77 (73.3\%) were confirmed on biopsy (without HHV-8 LAN-1 confirmation though); 11 (14.3\%) occurred in HIV-negative subjects. The majority $(63.6 \%)$ of the endemic KS cases was in the $\geq 50$ years of age group; the lesions were limited exclusively to the lower limbs, and all were males [5]. In our series of KS with unknown HIV serostatus, there were an almost equal number of males and females, with a similar mean age and distribution by histological stage as the documented HIVpositive KS. In terms of anatomical distribution, half were limited exclusively to the lower limbs. These data suggest that at least half of the patients with unknown HIV status were likely to be actually HIV infected. A Kenyan study has shown that, currently, endemic and HIV-related exhibit similar clinical presentation and natural history [25]. In an Ugandan study of 197 adults with HIV and KS, on the contrary, 55 percent were women; they were less likely to have lower limb lesions and more likely to have facial lesions than men. The authors suggested that gender affects the pathophysiology of KS [26].

\section{Conclusion}

The present study shows a relatively high discordance rate between the clinical and the biopsy-proven diagnosis of KS, indicating that "violaceous" skin lesions in an HIV endemic population warrant a biopsy. The relatively 


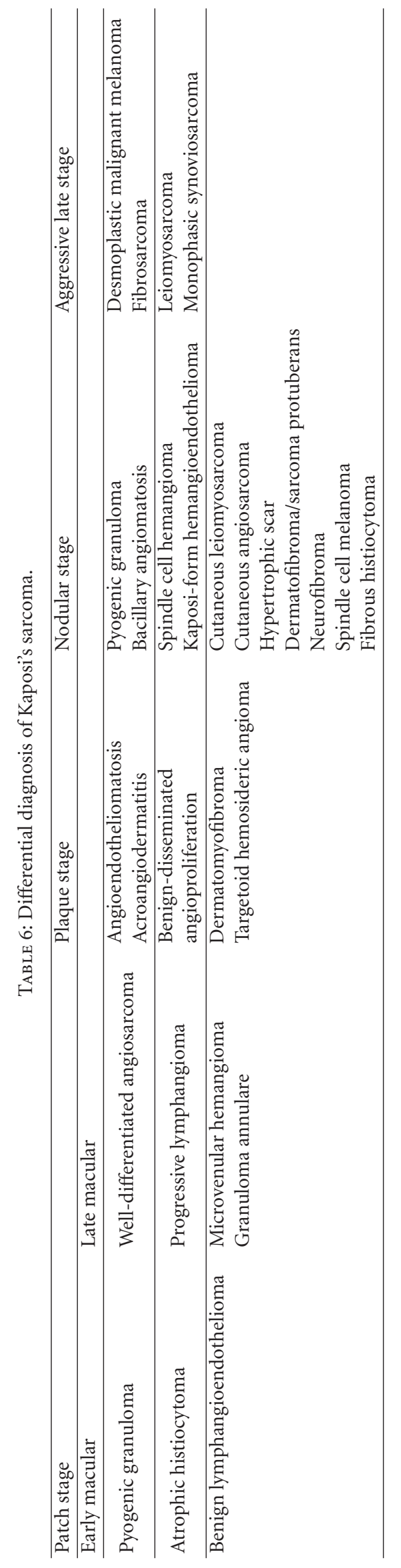


high histological discordance between the pre- and postimmunohistochemical diagnosis of KS warrants HHV-8 immunophenotyping to ascertain the diagnosis.

\section{Conflict of Interest}

The author declares that there is no conflict of interests.

\section{References}

[1] M. Aldenhoven, N. P. Barlo, and C. J. G. Sanders, “Therapeutic strategies for epidemic Kaposi's sarcoma," International Journal of STD \& AIDS, vol. 17, no. 9, pp. 571-578, 2006.

[2] S. M. Mbulaiteye, E. T. Katabira, H. Wabinga et al., "Spectrum of cancers among HIV-infected persons in Africa: the Uganda AIDS-Cancer Registry Match Study," International Journal of Cancer, vol. 118, no. 4, pp. 985-990, 2006.

[3] D. M. Parkin, "The global health burden of infectionassociated cancers in the year 2002," International Journal of Cancer, vol. 118, no. 12, pp. 3030-3044, 2006.

[4] E. Chokunonga, L. M. Levy, M. T. Bassett, B. G. Mauchaza, D. B. Thomas, and D. M. Parkin, "Cancer incidence in the African population of Harare, Zimbabwe: second results from the cancer registry 1993-1995," International Journal of Cancer, vol. 85, no. 1, pp. 54-59, 2000.

[5] G. Wamburu, E. J. Masenga, E. Z. Moshi, P. SchmidGrendelmeier, W. Kempf, and C. E. Orfanos, "HIV-associated and non-HIV associated types of Kaposi's sarcoma in an African population in Tanzania. Status of immune suppression and HHV-8 seroprevalence," European Journal of Dermatology, vol. 16, no. 6, pp. 677-682, 2006.

[6] J. L. Urquhart, A. Uzieblo, and S. Kohler, "Detection of HHV8 in pyogenic granuloma-like kaposi sarcoma," The American Journal of Dermatopathology, vol. 28, no. 4, pp. 317-321, 2006.

[7] "Mid-year population estimates," 2010, http://www.statssa .gov.za/.

[8] National Department of Health, National HIV Counseling and Testing Policy Guidelines, Department of Health, Pretoria, South Africa, 2010.

[9] Y. Chang, E. Cesarman, M. S. Pessin et al., "Identification of herpesvirus-like DNA sequences in AIDS-associated Kaposi's sarcoma," Science, vol. 266, no. 5192, pp. 1865-1869, 1994.

[10] N. Dupin, C. Fisher, P. Kellam et al., "Distribution of human herpesvirus-8 latently infected cells in Kaposi's sarcoma, multicentric Castleman's disease, and primary effusion lymphoma," Proceedings of the National Academy of Sciences of the United States of America, vol. 96, no. 8, pp. 4546-4551, 1999.

[11] E. J. Schwartz, R. F. Dorfman, and S. Kohler, "Human herpesvirus-8 latent nuclear antigen-1 expression in endemic kaposi sarcoma: an immunohistochemical study of 16 cases," The American Journal of Surgical Pathology, vol. 27, no. 12, pp. 1546-1550, 2003.

[12] R. M. Patel, J. R. Goldblum, and E. D. Hsi, "Immunohistochemical detection of human herpes virus-8 latent nuclear antigen-1 is useful in the diagnosis of Kaposi sarcoma," Modern Pathology, vol. 17, no. 4, pp. 456-460, 2004.

[13] W. Cheuk, K. O. Y. Wong, C. S. C. Wong, J. E. Dinkel, D. Ben-Dor, and J. K. C. Chan, "Immunostaining for human herpesvirus 8 latent nuclear antigen-1 helps distinguish kaposi sarcoma from its mimickers," American Journal of Clinical Pathology, vol. 121, no. 3, pp. 335-342, 2004.
[14] J. A. Ramirez, W. B. Laskin, and J. Guitart, "Lymphangiomalike Kaposi sarcoma," Journal of Cutaneous Pathology, vol. 32, no. 4, pp. 286-292, 2005.

[15] P. Pyakurel, F. Pak, A. R. Mwakigonja, E. Kaaya, T. Heiden, and P. Biberfeld, "Lymphatic and vascular origin of Kaposi's sarcoma spindle cells during tumor development," International Journal of Cancer, vol. 119, no. 6, pp. 1262-1267, 2006.

[16] W. Grayson and L. Pantanowitz, "Histological variants of cutaneous Kaposi sarcoma," Diagnostic Pathology, vol. 3, no. 1, pp. 31-48, 2008.

[17] P. J. O’Donnell, L. Pantanowitz, and W. Grayson, "Unique histologic variants of cutaneous Kaposi sarcoma," The American Journal of Dermatopathology, vol. 32, no. 3, pp. 244-250, 2010.

[18] R. Russell Jones, G. Orchard, B. Zelger, and E. Wilson Jones, "Immunostaining for CD31 and CD34 in Kaposi sarcoma," Journal of Clinical Pathology, vol. 48, no. 11, pp. 1011-1016, 1995.

[19] K. M. Chu, G. Mahlangeni, S. Swannet, N. P. Ford, A. Boulle, and G. Van Cutsem, "AIDS-associated Kaposi's sarcoma is linked to advanced disease and high mortality in a primary care HIV programme in South Africa," Journal of the International AIDS Society, vol. 13, no. 1, pp. 23-27, 2010.

[20] N. Kumarasamy, K. K. Venkatesh, B. Devaleenol, S. Poongulali, and N. Ahilasamy, "Regression of Kaposi's sarcoma lesions following highly active antiretroviral therapy in an HIVinfected patient," International Journal of STD \& AIDS, vol. 19, no. 11, pp. 786-788, 2008.

[21] P. A. Agaba, H. M. Sule, R. O. Ojoh et al., "Presentation and survival of patients with AIDS-related Kaposi's sarcoma in Jos, Nigeria," International Journal of STD \& AIDS, vol. 20, no. 6, pp. 410-413, 2009.

[22] E. Calonje, "Vascular tumors: tumors and tumor-like conditions of blood vessels and lymphatics," in Lever's Histopathology of the Skin, D. Elder, Ed., pp. 1007-1056, Wolters/ Kluwer/Lippincott Williams \& Wilkins, Philadelphia, Pa, USA, 10th edition, 2009.

[23] T. Mentzel and H. Kutzner, "Haemorrhagic dermatomyofibroma (plaque-like dermal fibromatosis): clinicopathological and immunohistochemical analysis of three cases resembling plaque-stage Kaposi's sarcoma," Histopathology, vol. 42, no. 6 , pp. 594-598, 2003.

[24] D. A. Wada, S. L. Perkins, S. Tripp, C. M. Coffin, and S. R. Florell, "Human herpesvirus 8 and iron staining are useful in differentiating Kaposi sarcoma from interstitial granuloma annulare," American Journal of Clinical Pathology, vol. 127, no. 2, pp. 263-270, 2007.

[25] O. W. Mwanda, P. Fu, R. Collea, C. Whalen, and S. C. Remick, "Kaposi's sarcoma in patients with and without human immunodeficiency virus infection, in a tertiary referral centre in Kenya," Annals of Tropical Medicine and Parasitology, vol. 99, no. 1, pp. 81-91, 2005.

[26] W. Phipps, F. Ssewankambo, H. Nguyen et al., "Gender differences in clinical presentation and outcomes of epidemic kaposi sarcoma in Uganda," PLoS ONE, vol. 5, no. 11, Article ID e13936, 2010. 


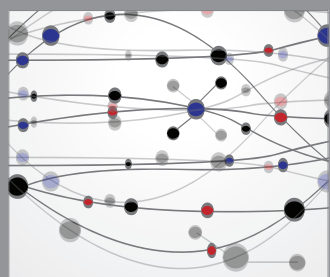

The Scientific World Journal
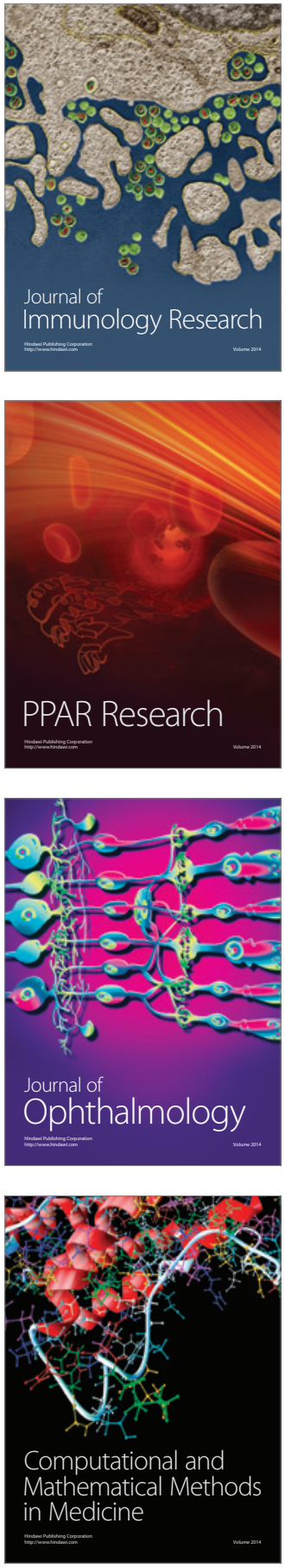

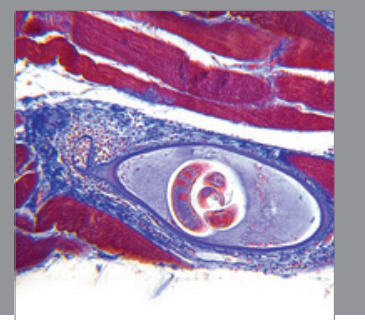

Gastroenterology

Research and Practice
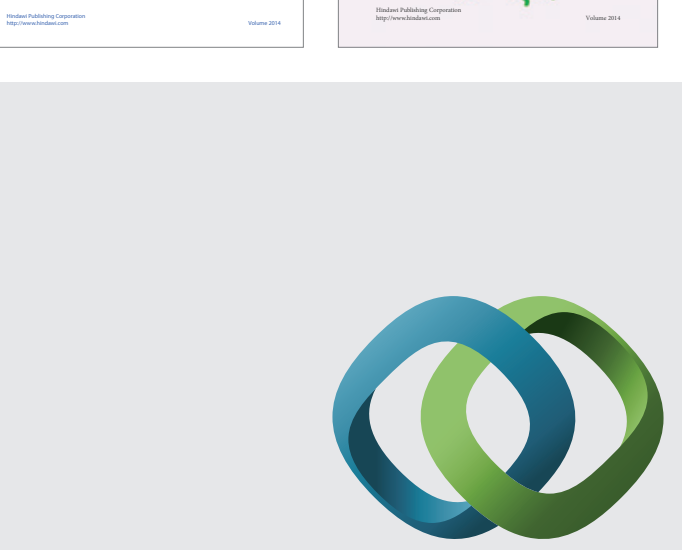

\section{Hindawi}

Submit your manuscripts at

http://www.hindawi.com
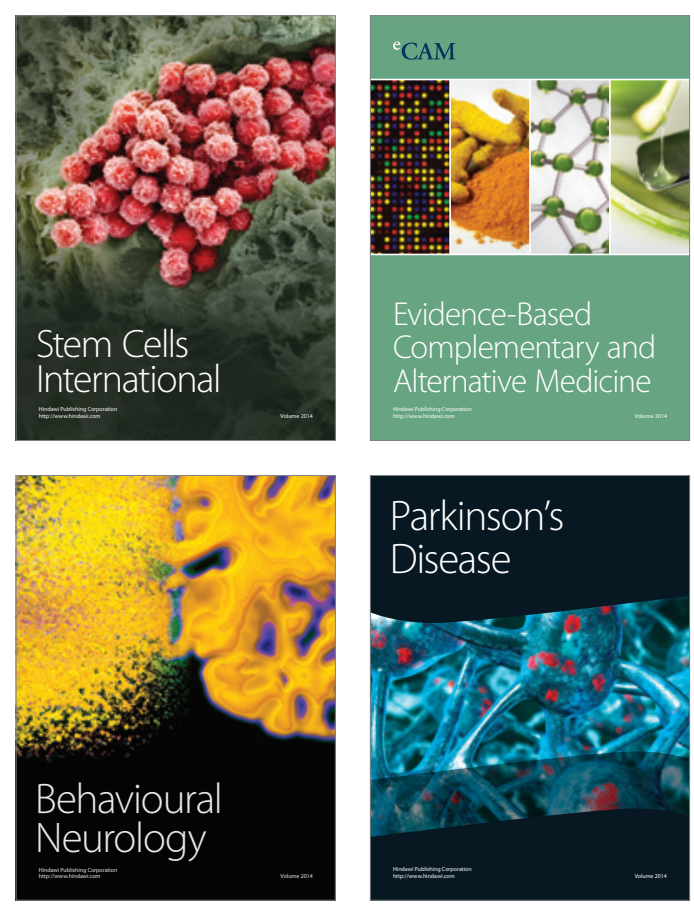

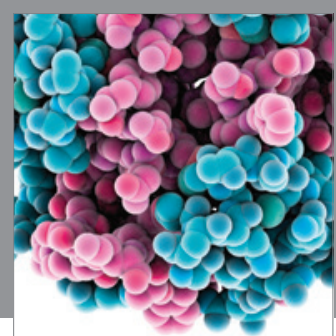

Journal of
Diabetes Research

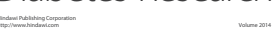

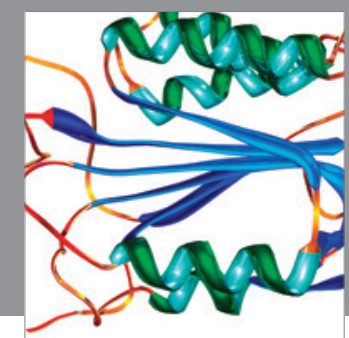

Disease Markers
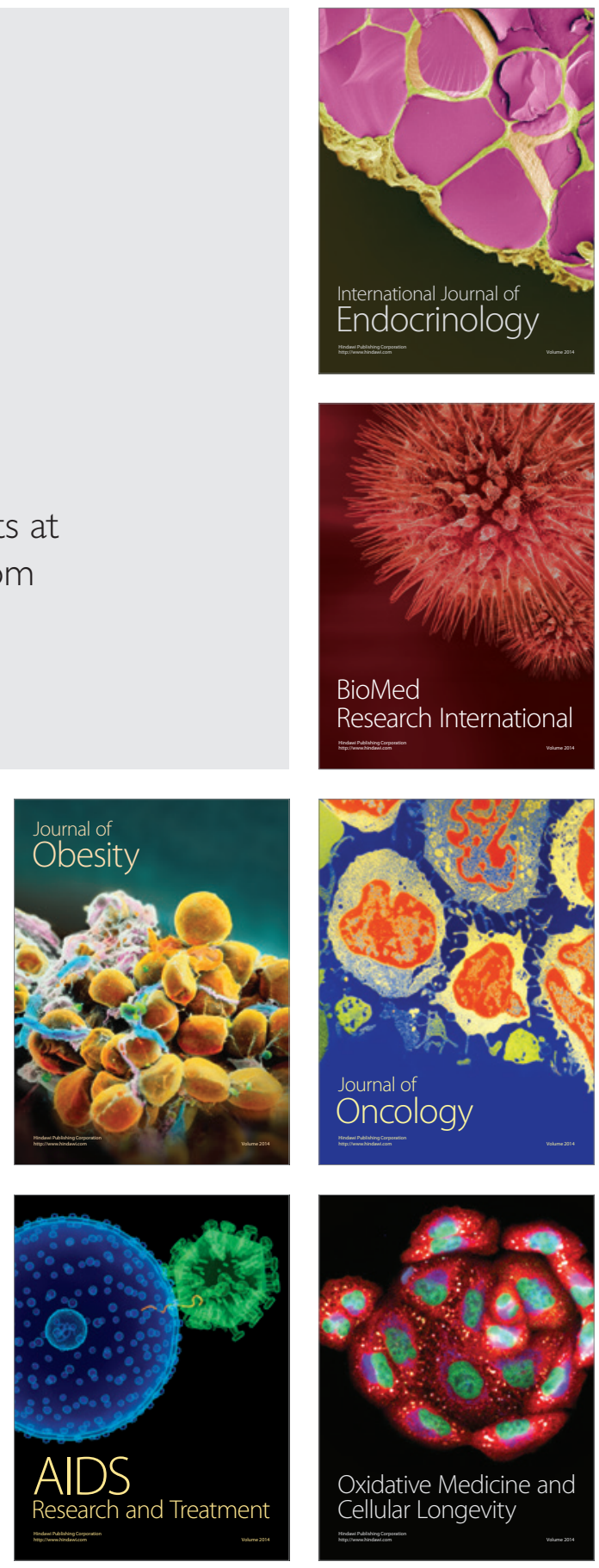